\title{
Reverse translated and gold standard continuous performance tests predict global cognitive performance in schizophrenia
}

\author{
Andrew W. Bismark², ${ }^{1,2}$ Michael L. Thomas², Melissa Tarasenko' ${ }^{1}$ Alexandra L. Shiluk², Sonia Y. Rackelmann², \\ Jared W. Young ${ }^{1,2}$ and Gregory A. Light ${ }^{1,2}$
}

\begin{abstract}
Attentional dysfunction contributes to functional impairments in schizophrenia (SZ). Sustained attention is typically assessed via continuous performance tasks (CPTs), though many CPTs have limited cross-species translational validity and place demands on additional cognitive domains. A reverse-translated 5-Choice Continuous Performance Task (5C(PT) for human testing - originally developed for use in rodents - was designed to minimize demands on perceptual, visual learning, processing speed, or working memory functions. To-date, no studies have validated the 5C-CPT against gold standard attentional measures nor evaluated how 5C-CPT scores relate to cognition in SZ. Here we examined the relationship between the 5C-CPT and the CPT-Identical Pairs (CPT-IP), an established and psychometrically robust measure of vigilance from the MATRICS Consensus Cognitive Battery (MCCB) in a sample of SZ patients $(n=35)$. Relationships to global and individual subdomains of cognition were also assessed. 5C-CPT and CPT-IP measures of performance (d-prime) were strongly correlated $(r=0.60)$. In a regression model, the 5C-CPT and CPT-IP collectively accounted for $54 \%$ of the total variance in MCCB total scores, and $27.6 \%$ of overall cognitive variance was shared between the 5C-CPT and CPT-IP. These results indicate that the reverse translated 5C-CPT and the gold standard CPTIP index a common attentional construct that also significantly overlaps with variance in general cognitive performance. The use of simple, cross-species validated behavioral indices of attentional/cognitive functioning such as the 5C-CPT could accelerate the development of novel generalized pro-cognitive therapeutics for SZ and related neuropsychiatric disorders.
\end{abstract}

\section{Introduction}

Attentional dysfunction is a core deficit in schizophrenia (SZ) that negatively impacts functional outcomes ${ }^{1}$. Current pharmacological treatments, however, have limited efficacy for treating cognitive deficits. The link between cognition and functional outcomes, combined with lack-of-effective treatments, has galvanized research to identify pro-cognitive therapeutics for SZ

Correspondence: Jared W Young (jaredyoung@ucsd.edu)

${ }^{1}$ VISN-22 Mental IIIness Research Education and Clinical Center (MIRECC), VA San Diego Healthcare System, San Diego, USA

${ }^{2}$ Department of Psychiatry, University of California, San Diego, USA patients $^{2-6}$. While pre-clinical research has yielded insights that may inform the future development of procognitive treatments, profound translational gaps across pre-clinical and clinical studies exist. These gaps remain in large part due to the limited use of cognitive paradigms with cross-species translational validity and relevance ${ }^{7-9}$.

Over the past 15 years the National Institutes of Mental Health (NIMH) has sponsored two projects intended to improve the measurement of cognitive deficits in SZ and identify promising tasks with translational validity. The first, the Measurement and Treatment Research to Improve Cognition in Schizophrenia (MATRICS) 
initiative, was an effort to standardize cognitive measurement for approval of any pro-cognitive compounds. The product of this initiative, the MATRICS Consensus Cognitive Battery (MCCB), was designed for use in clinical trials and now represents the "gold standard" assessment of cognitive functioning in SZ. Given that the purpose of this battery was to inform clinical trials, limited effort was made to develop this battery in conjunction with cross-species available non-human tests ${ }^{10}$. The Cognitive Neuroscience Treatment Research to Improve Cognition in Schizophrenia (CNTRICS) initiative was established to identify cross-species relevant behavioral paradigms of cognitive constructs such as attention ${ }^{11}$.

The 5-choice continuous performance test (5C-CPT) was developed for use in mice ${ }^{10}$, and was highlighted by the CNTRICS initiative as a promising cross-species paradigm for assessing the control of attention ${ }^{11}$. The $5 \mathrm{C}$ CPT has shown good cross-species validity, including (a) $36 \mathrm{~h}$ sleep deprivation-induced deficits ${ }^{12}$; (b) amphetamine-induced improvement ${ }^{13}$; and (c) vigilance decrement observations across time ${ }^{6,10}$. Importantly, patients with SZ exhibit deficient 5C-CPT performance ${ }^{6,14}$, consistent with other $\mathrm{CPTs}^{15,16}$. To-date however, no studies have validated the $5 \mathrm{C}$-CPT against a consensus gold standard attentional measurement nor evaluated how $5 \mathrm{C}-\mathrm{CPT}$ performance is related to cognition in SZ.

Continuous performance tasks (CPTs) are the most common paradigms used for quantifying attentional functioning in neuropsychiatric patients including $\mathrm{SZ}^{15}$. In their simplest form, CPTs involve subjects' being presented with a string of rapidly occurring stimuli and asked to identify targets from among background or non-target stimuli. Common variants of the CPT include CPTIdentical Pairs version (CPT-IP- chosen as part of the $\mathrm{MCCB}$ ), degraded stimulus CPT (DS-CPT), the AX-CPT (AX-CPT), and the Connors' CPT (C-CPT). Whereas, all $\mathrm{CPTs}$ are designed to quantify sustained attention/vigilance, each CPT emphasizes a unique balance between target detection and response inhibition, which may have important implications for cognition. Important methodological differences also exist across tasks and place demands on other cognitive domains ${ }^{17}$. For example, the CPT-IP has been referred to as a "memory load" CPT given that responses are required when two sequentially presented numbers are identical, likely indexing both attention and working memory. The DS-CPT has been referred to as a "perceptual load CPT" since target stimuli with varying degrees of degradation are presented to subjects and therefore is sensitive to both perceptual and attentional deficits ${ }^{18,19}$. The AX-CPT requires responding when the letter $\mathrm{X}$ follows an $\mathrm{A}$ in sequence (ignoring $\mathrm{B}$ and $\mathrm{Y}$ stimuli presentations), likely requiring modest working memory functioning in addition to attentional control $^{20}$. In contrast, the Connors' CPT simply requires responses when target stimuli (letters other than $\mathrm{X}$ ) are presented, and response inhibition when non-target stimuli (letter X) are presented ${ }^{21}$. Despite these task differences, CPT paradigms have demonstrated clinical sensitivity in quantifying attentional deficits in first episode and chronic SZ patients, unmedicated SZ patients, and unaffected first-degree relatives of SZ patients ${ }^{19,22-25}$.

Psychometric theories of cognition suggest that cognitive abilities are hierarchically structured such that variance (and covariance) in specific abilities can be explained by a smaller number of general abilities ${ }^{26}$. Attention is thought to represent a core domain of cognitive functioning, which might explain large correlations between measures of attentional performance and overall cognition as measured by the $\mathrm{MCCB}^{27}$. Furthermore, attention-dependent cognitive measures have been used as exemplars to demonstrate applicability as important targets of medications used to treat $\mathrm{SZ}^{2}$.

Although, CPTs are established measures of attentional functioning, they are often multidimensional (as described above), potentially confounding attentional dysfunction with deficits in perceptual or other cognitive domains, e.g., working memory. Moreover, lack-of-cross-species CPTs further limits opportunities for novel treatment development. Quantifying attentional abilities underlying normal and impaired cognitive performance in SZ patients on the $5 \mathrm{C}-\mathrm{CPT}$ could accelerate the development of pro-cognitive therapeutics. The availability of tasks for measuring similar attentional abilities across species would enable researchers to develop agents for targeting attentional systems in animals and begin spanning the translational gap to human findings.

This study aimed to characterize SZ patients' attentional task performance on the reverse-translated $5 \mathrm{C}-\mathrm{CPT}$ in comparison to a "gold standard" measure, the CPT-IP, and to assess its relationship with a global measure of cognition (MCCB). We hypothesized that measures of 5C-CPT and CPT-IP task performance would be significantly correlated and that attentional functioning (as measured by both CPTs) would predict cognitive test performance (MCCB total score). To determine the effects of symptom severity on attentional and cognitive functioning, we assessed the extent to which $5 \mathrm{C}-\mathrm{CPT}$, CPT-IP, and MCCB scores were related to positive and negative symptom ratings.

\section{Methods \\ Participants}

Thirty-five SZ patients between the ages of 18-61 years old were recruited from a transitional care facility that primarily serves people with diagnoses of SZ or schizoaffective disorder (Table 1). Exclusion criteria for the study included: history of neurological disease, history of 
Table 1 Participant demographics

\begin{tabular}{ll}
\hline Demographics ( \pm s.d., $\mathbf{m i n}-\mathbf{m a x})(\boldsymbol{n}=\mathbf{3 5})$ & \\
\hline Mean age (yrs) & $36.1( \pm 12.7,19-61)$ \\
Education & $12.1( \pm 2.1,8-18)$ \\
Sex (\% male) & $51.0 \%$ \\
Smoking & $0 \%^{\mathrm{a}}$ \\
Right handedness & $63.9 \%$ \\
Age of onset (yrs) & $19.3( \pm 4.5,8-30)$ \\
Illness duration (yrs) & $16.7( \pm 12.9,1-47)$ \\
SAPS total score & $5.14( \pm 4.7,0-16)$ \\
SANS total score & $6.43( \pm 4.2,0-16)$ \\
\hline
\end{tabular}

${ }^{\mathrm{a}}$ All participants were housed within a non-smoking transitional care facility, and were free from nicotine for at least 2 months prior to testing. Although, metaanalytic research demonstrated substantial comorbidity between SZ and nicotine use $\mathrm{e}^{46}$ more recent research indicated smoking may decrease MCCB performance ${ }^{47}$

major head injury (loss-of-consciousness $>15 \mathrm{~min}$ ), substance dependence within the last 6 months, severe systemic medical illness (e.g., Hepatitis C, HIV, insulindependent diabetes), IQ below 70 as estimated by the reading subtest of the Wide Range Achievement Test (WRAT), and difficulty with hearing, vision or English language comprehension that may interfere with the patient understanding consent, screening questions, and task directions. The Institutional Review Board of University of California, San Diego, approved all experimental procedures (IRB\#130874). All participants underwent an informed consent procedure, structured clinical diagnostic assessments including a modified Structured Clinical Interview for DSM-IV Axis I disorders (SCID-I), and the Scales for the Assessment of Positive and Negative Symptoms (SAPS and SANS) ${ }^{28,29}$, and cognitive assessment using the MCCB (the Mayer-Salovey-Caruso Emotional Intelligence Test was not administered due to time limitations). The CPT-IP score was omitted when calculating the MCCB total score to avoid biasing predictive relationships. The MCCB neurocognitive composite score was calculated using the mean of the domain $T$ scores, as is consistent with prior publications ${ }^{30}$. The $5 \mathrm{C}$ $\mathrm{CPT}$ was completed following the diagnostic and cognitive assessments.

\section{5-choice continuous performance task (5C-CPT)}

The $5 \mathrm{C}-\mathrm{CPT}$ requires responses to targets and inhibition of responses to non-target trials. Participants were instructed to move the joystick in the direction a circle (target stimuli) appears, but inhibit from responding if five circles appeared simultaneously (non-target stimuli) (Fig. 1a) ${ }^{14}$. Stimuli were presented for $100 \mathrm{~ms}$ in a random order to reduce temporal predictability with a $1 \mathrm{~s}$ response window available and a variable inter-trial interval (ITI; $0.5,1.0$, or $1.5 \mathrm{~s}$ ). All participants performed a practice block prior to initiating the session and indicated they understood the task. The full task consisted of 270 trials, 225 target and 45 non-target stimuli, presented pseudorandomly so that no $>3$ presentations of a specific stimulus appeared consecutively. Responses were recorded and include hits and misses to target trials, and false alarms (FAs) and correct rejections (CRs) to non-target trials. Composite metrics of task performance were used in the analysis of performance, including hit rate (HR), false alarm rate (FAR), task accuracy, $\mathrm{d}^{\prime}$, and responsivity index (RI) as indicated in our previous work ${ }^{6}$.

\section{Continuous performance task-identical pairs version (CPT- IP)}

Developed by Cornblatt et al. ${ }^{31}$, the CPT-IP version is a computerized measure of sustained, focused attention. The task involves monitoring a series of numbers $(2,3$, or 4 digits in length) and responding when two identical stimuli occur consecutively (Fig. 1b).

\section{Statistical analyses}

All statistical analyses were conducting using SPSS (IBM Corp., Armonk, NY, USA). Paired sample $t$-tests were used to compare performance metrics between $5 \mathrm{C}$ $\mathrm{CPT}$ and CPT-IP. Pearson correlations were used to test relationships between $\mathrm{CPT} \mathrm{d}^{\prime}$ measures, MCCB total score, and symptom ratings (SANS, SAPS). Multivariate linear regression models using $5 \mathrm{C}-\mathrm{CPT}$ and CPT-IP $\mathrm{d}^{\prime}$ measures as predictors, were used to determine the unique contribution of each behavioral measure to cognition (MCCB total score). Estimates of variance explained $\left(R^{2}\right)$, standardized regression slopes $(\beta)$, and Pearson correlations between predictors are reported ${ }^{32}$. Statistical significance for correlations was adjusted for multiple comparisons using the Bonferroni method ${ }^{33,34}$.

\section{Results}

As shown in Table 2, SZ patients exhibited poorer performance on the CPT-IP, demonstrated by significantly lower hit rate (HR), higher false alarm rate (FAR), and lower $\mathrm{d}^{\prime}$ compared to $5 \mathrm{C}-\mathrm{CPT}$ performance. There was however, a large positive correlation between $5 \mathrm{C}-\mathrm{CPT}$ and CPT-IP $\mathrm{d}^{\prime}$ measures $(r=0.60, p<0.001)$. Additional correlations between each CPT d', MCCB total score and MCCB subscales are reported in Table 3. The $5 \mathrm{C}-\mathrm{CPT} \mathrm{d}^{\prime}$ was significantly and positively correlated with MCCB composite scores, as well as with working memory (WM) and reasoning and problem solving (RPS) subdomain scores, with a trend-level positive relationship with the verbal learning subdomain. The CPT-IP $\mathrm{d}^{\prime}$ was significantly correlated with MCCB composite score and all cognitive subdomain scores except verbal learning 


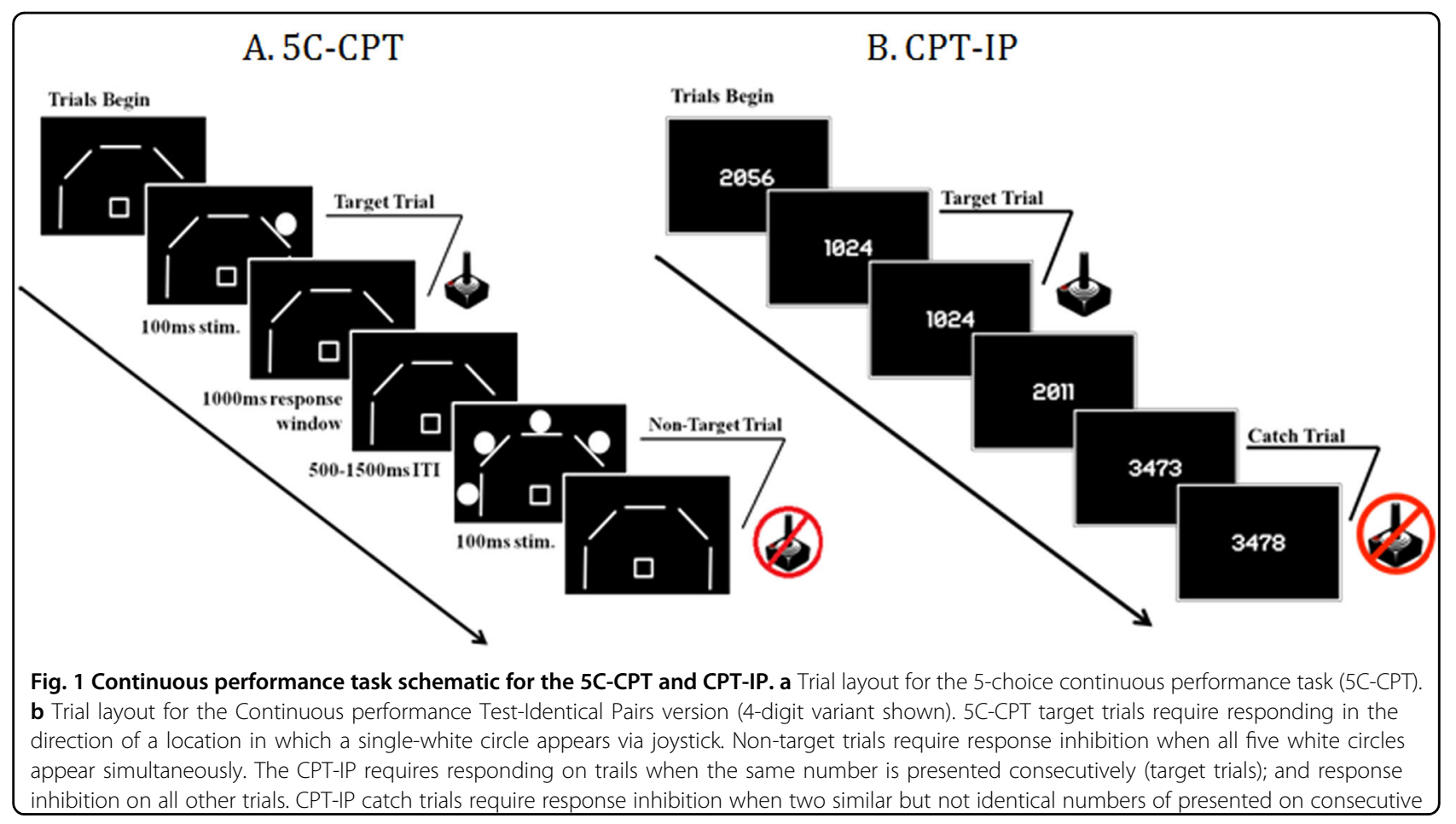

\section{Table 2 Behavioral task descriptive statistics}

\begin{tabular}{lll}
\hline Task/measure & \multicolumn{2}{l}{ Mean (SEM) } \\
\cline { 2 - 3 } & 5C-CPT & CPT-IP \\
\hline $\mathrm{d}^{\prime}$ & $3.85(0.23)$ & $1.85(0.13)^{\mathrm{a}}$ \\
Hit rate (HR) & $0.90(0.35)$ & $0.68(0.04)^{\mathrm{a}}$ \\
False alarm rate (FAR) & $0.03(0.01)$ & $0.15(0.01)^{\mathrm{a}}$ \\
Responsivity index (RI) & $-0.23(0.07)$ & $-0.24(0.05)$ \\
MCCB (T-scores) & Mean (SEM) & Range \\
Composite score & $34.29(1.2)$ & $18.8-49.4$ \\
Speed of processing & $30.8(1.8)$ & $8-55$ \\
Visual learning & $31.5(1.9)$ & $14-59$ \\
Verbal learning & $34.1(0.9)$ & $21-46$ \\
Working memory & $33.3(2.2)$ & $5-55$ \\
Reasoning and problem solving & $41.7(1.4)$ & $28-59$ \\
\hline
\end{tabular}

Behavioral task performance and MCCB composite and subscale means, standard errors, and response ranges

andicates $p<0.01$

(Table 3). There were weak but non-significant correlations between SANS and SAPS scores and 5C-CPT, CPT-IP, or MCCB total scores (all $r$ 's $<0.31$, ps $>0.08$ ) (Table 3), consistent with previous reports ${ }^{19}$.

\section{Regression analyses}

To assess the shared and unique contributions of each $\mathrm{CPT}$ to cognition, MCCB composite scores were
Table 3 Attention-cognition correlations

\begin{tabular}{|c|c|c|c|}
\hline 5C-CPT & $5 \mathrm{C}-\mathrm{CPT}$ & $\begin{array}{l}\text { CPT-IP } \\
0.60^{*}\end{array}$ & $\begin{array}{l}\text { Fisher's z } \\
p \text {-values }\end{array}$ \\
\hline MCCB composite & $0.54^{*}$ & $0.72^{*}$ & NS \\
\hline Speed of processing & 0.33 & $0.56^{*}$ & $0.03^{*}$ \\
\hline Working memory & $0.54^{*}$ & $0.64^{*}$ & NS \\
\hline Verbal learning & $0.44+$ & 0.41 & NS \\
\hline Visual learning & 0.26 & $0.52^{*}$ & $0.02 *$ \\
\hline Reasoning and problem solving & $0.51 *$ & $0.54^{*}$ & NS \\
\hline SANS total score & -0.30 & -0.15 & NS \\
\hline SAPS total score & -0.28 & -0.21 & NS \\
\hline
\end{tabular}

* indicates $p<.004$

Pearson correlations between 5C-CPT and CPT-IP d's, MCCB composite (without the CPT-IP included), subscale T-scores, and SANS and SAPS total scores. Statistical significance $\left.{ }^{*}\right)$ was determined based on a Bonnferoni correction, which required $p<0.004$. Plus symbol $(+)$ indicates corrected trend-level significance $p<0.01$. Right column depicts $p$-values for Fisher-z correlation comparisons between left and middle columns using single-sided testing

\section{presentations}

regressed onto $5 \mathrm{C}-\mathrm{CPT}$ and $\mathrm{CPT}-\mathrm{IP} \mathrm{d}^{\prime}$ scores. A model including both CPTs accounted for $53.9 \%$ of the variance in MCCB composite score $\left(F_{(2,32)}=18.7, p<0.001\right)$. Comparisons of model parameter estimates (standardized regression slopes) indicated that $27.6 \%$ of the variance in global MCCB performance was shared attentional variance between the 5C-CPT and CPT-IP. The 5C-CPT further accounted for a unique but non-significant $2.0 \%$ of 


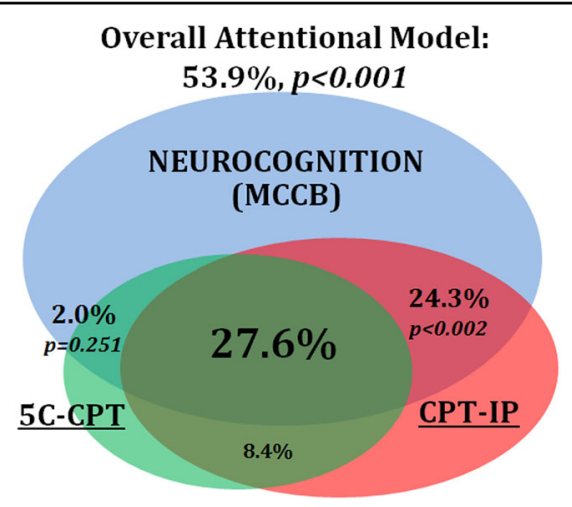

Fig. 2 Behavioral measure variance components predicting cognition. Total model variance in cognition (MCCB Total score) accounted for was 53.9\%. Outer circles depict the unique variance proportions for each predictor. The CPT-IP accounted for $24.3 \%$ of the variance, while the 5C-CPT uniquely accounted for only $2.0 \%$. The variance shared between the 5 C-CPT and the CPT-IP accounted for $27.6 \%$ of the variance in cognition

the variance in cognition $(b=0.956, \beta=0.175, p=0.251)$, while the CPT-IP uniquely accounted for an additional $24.3 \%$ of the variance $(b=5.62, \beta=0.616, p<0.001)$ in $\mathrm{MCCB}$ composite scores. A Venn diagram visualizing the relationships among the three variables is shown in Fig. 2.

\section{Discussion}

The present study validates the $5 \mathrm{C}-\mathrm{CPT}$ as an attentional measure relevant to cognition in SZ through its strong association with the gold standard CPT-IP as indicated by their combined relationship with cognitive test performance, independent of symptomatology. The strong relationship observed between the $5 \mathrm{C}-\mathrm{CPT}$ and the CPT-IP $(r=0.60)$, and whose combined variance accounted for more than half of the variance MCCB total scores (53.9\%), underscores the importance of attentional functioning to cognition. Regression analyses indicated that, of the $36 \%$ of variance shared between the $5 \mathrm{C}-\mathrm{CPT}$ and the CPT-IP, $27.6 \%$ is likely attention-specific variance that contributes to global cognition (Fig. 2). The residual, non-attentional variance shared between the $5 \mathrm{C}-\mathrm{CPT}$ and CPT-IP (8.4\%) may partially represent behavioral effort required to complete these tasks ${ }^{35}$, or method variance shared between CPTs not associated with cognition. The considerable variance shared between $5 \mathrm{C}-\mathrm{CPT}, \mathrm{CPT}$-IP, and MCCB scores likely reflects an attentional construct measured to differing degrees by all three tasks.

The 5C-CPT primarily measures attentional functioning similarly to the CPT-IP but with important differences. This is evidenced by the differing relationships between each CPT and the MCCB composite score. After the $27 \%$ variance shared between the CPTs and $\mathrm{MCCB}$ was accounted for, the residual $2.0 \%$ of shared variance between $5 \mathrm{C}-\mathrm{CPT}$ and $\mathrm{MCCB}$ was no longer significant $(p=0.25)$ (Fig. 2). This finding represents a strength of the $5 \mathrm{C}-\mathrm{CPT}$ as it suggests that the measure may provide a relatively more specific index of attention compared to the gold-standard CPT-IP. The CPT-IP, in contrast, accounted for an additional 24.3\% $(p<0.001)$ of the variance in MCCB performance after accounting for shared variance with $5 \mathrm{C}-\mathrm{CPT}$, indicating that $\mathrm{CPT}$-IP performance may reflect not only attentional ability but also broader cognitive functioning. This finding is further supported by significant correlations between CPT-IP performance and MCCB subdomains of speed of processing and visual learning (Table 3). The relationships among these domains with CPT-IP performance likely reflect the additional CPT-IP task demands of processing rapidly presented numerical strings and evaluating each as a potential target for comparison to recently presented stimuli. The cognitive resources required for rapid evaluation and matching of numerical strings likely involves resources beyond attention and are thus reflected in the non-attentional relationship between CPT-IP and neurocognition. In contrast, the $5 \mathrm{C}-\mathrm{CPT}$ does not place such demands on speed of processing, likely reducing its link to this domain. As the relationships between symptom ratings and attentional or cognitive test performance did not survive correction, it is thought current symptom levels minimally affected the relationship between CPT and MCCB performance.

The direct comparison of $5 \mathrm{C}$-CPT and CPT-IP performance enables cross-task validation the $5 \mathrm{C}-\mathrm{CPT}$ compared to the gold standard CPT-IP. Patient performance across all 5C-CPT performance metrics (HR, FAR, d') were significantly better than those of the CPT-IP, suggesting that $5 \mathrm{C}-\mathrm{CPT}$ may generally be less difficult than CPT-IP. The CPT-IP explicitly manipulates task difficulty across blocks using two-, three-, or four-digit strings, compared to the single difficulty level of the 5C-CPT. Supplementary analyses revealed a linear decrease in $\mathrm{d}^{\prime}$ with increased difficulty on the CPT-IP, consistent with prior reports ${ }^{36}$. The CPT-IP two-digit condition, compared to the three- and four-digit conditions, was most strongly correlated with $5 \mathrm{C}$-CPT, suggesting that this condition most closely approximates the difficulty of the 5C-CPT (Supplemental figure S1). To test the specificity of the relationship between the CPT-IP-2-digit version and the $5 \mathrm{C}$-CPT, all statistics were rerun using only the CPT-IP-2-digit version. the overall model and relationships between cognition and each CPT did not significantly change (Supplemental Material). A recent study comparing four versions of the CPT-IP found no increased sensitivity between versions in SZ patients ${ }^{36}$. Therefore, we aggregated scores across CPT-IP difficulty levels to provide a task-level metric of attentional functioning to compare to the single difficulty of the $5 \mathrm{C}-\mathrm{CPT}$. Regardless of this difference in score calculation, the 
CPT-IP and 5C-CPT shared significant variance and were both significantly related to MCCB performance, underscoring their shared measurement of attention and its relationship to cognition. The current results demonstrate the reverse translated $5 \mathrm{C}-\mathrm{CPT}$ measures attentional functioning similarly to the gold standard CPT-IP, and can significantly predict cognition in SZ patients in a real world treatment setting.

Attentional functioning (in both CPTs) was also significantly correlated with other cognitive subdomains, and outlines attentional functioning as a necessary but insufficient construct underlying global cognition. Recent research has indicated the importance of attentional functioning for other cognitive processes (i.e., processing speed, planning, reasoning, and problem solving), as well as its direct and indirect relationships with functional outcomes in $\mathrm{SZ}^{11,37,38}$. The current data suggest good convergent validity among CPTs for attention measures, but also highlights the challenge of measuring a cognitive domain in isolation. The pattern of correlations between the $5 \mathrm{C}-\mathrm{CPT}, \mathrm{CPT}-\mathrm{IP}$, and $\mathrm{MCCB}$ subdomains indicates increased specificity in attentional measurement between the $5 \mathrm{C}-\mathrm{CPT}$ and cognition compared to the gold standard CPT-IP. In contrast, correlations between CPTs and other cognitive domains ranged from small to moderate (Table 3). Recent neuropsychological findings in adult SZ patients indicate potential gender effects for attention and reasoning/problem solving specifically ${ }^{39}$. While the current study lacked the power to investigate gender specific effects on cognition, they may help explain the moderate correlations between the $5 \mathrm{C}-\mathrm{CPT}$ and the reasoning and problem-solving subdomain. The relationship between attention and other cognitive subdomains in the current study was present in both CPTs, most notably with WM. Recent research has indicated a detrimental interaction between WM and attention where SZ patients demonstrate disproportional attentional impairment under increasing WM load compared to healthy controls ${ }^{40}$. Additionally, deficits in attention and WM may also be the product of a narrowing of attentional focus compared to controls, accounting for reported deficits in both domains ${ }^{41}$. As SZ patients display deficits across cognitive domains including WM and attentional functioning, the challenge persists on how to accurately quantify attentional functioning in the absence of other cognitive measures. Unfortunately, common CPT variants (CPT-IP, DS-CPT, AX-CPT, Connor's CPT) all suffer from various levels of interpretive ambiguity by including additional cognitive task demands in the measurement of attentional functioning ${ }^{2,17}$. As attention is thought to be a necessary component of higher-order cognitive functioning, inter-correlations amongst related cognitive domains are to be expected. Thus, future research should utilize reverse-translated tasks targeting specific cognitive domains to better disentangle specific cognitive deficits in psychiatric patients. Given the availability of the mouse ${ }^{42}$ and rat $^{43,44} 5 \mathrm{C}$-CPT versions, treatments that improve task performance in animals could potentially be used to improve global cognitive functioning in humans.

A few study limitations deserve discussion, most notably the lack of a non-psychiatric comparison group. Future studies will investigate both SZ and healthy control samples to ascertain differential behavioral relationships between CPT task performance and cognitive functioning across groups. Additionally, as in the vast majority of SZ studies, all participants were medicated at the time of testing, with most treated on a combination of typical and atypical antipsychotic mediation along with other psychotropics. Although, the heterogeneity of medication regimens in our cross-sectional sample precluded the examination of medication effects, our behavioral measures were still sensitive to cognition with this medication-heterogeneous sample. Nonetheless, we cannot rule out an impact of antipsychotic medications on our findings, and future randomized controlled trials should prospectively examine medication effects on subjects. Finally, it is possible that the overlapping variance between $5 \mathrm{C}-\mathrm{CPT}$ and CPT-IP represents a latent construct other than attention that is relevant to cognitive test performance in SZ. For example, these tasks may also provide a measurement of cognitive control that has been proposed to be consistently disturbed across psychiatric conditions $^{45}$. Beyond cognitive control, however, given the specific task demands of the $5 \mathrm{C}-\mathrm{CPT}$ and low correlations with non-attentional MCCB subdomains, it is unlikely that other cognitive abilities account for such high-overlapping variance.

In conclusion, we demonstrate a novel reverse translated behavioral measure of attention is robustly related to an established measure of attention and cognitive test performance in a group of transitionally housed SZ inpatients. By validating novel reverse-translated laboratory measures like the $5 \mathrm{C}-\mathrm{CPT}$, together with existing gold standard measures of attention and cognition, we can provide more direct cross-species relationships to aid the development of pro-cognitive therapeutics, and ultimately improve functional outcomes in $\mathrm{SZ}^{5}$. This translational approach may provide further utility by identifying individuals likely to benefit from treatment and identifying those who may benefit from additional targeted pharmacological or psychosocial pre-treatments to help boost treatment gains and long-term functional outcomes. Finally, as attentional deficits may be present prior to full disease onset and signal poor functional outcomes, this approach may facilitate early identification of individuals at elevated risk for developing pathologies with primary attentional dysfunction. 


\section{Acknowledgements}

We would like to thank Mrs. Joyce Sprock, Ms. Wendy Zhang for their aid in the studies. We would also like to thank all of the patients and their families for their aid in participation. This work was supported by the Sidney R. Baer Jr. Foundation, Brain and Behavioral Research Foundation, the Veteran's Administration VISN 22 Mental IIIness Research, Education, and Clinical Center and NIH grants R01-MH104344, UH2-MH109168, K23 MH102420. Dr. J.W.Y. has received funding from the Cerca Insights and Lundbeck Ltd, and has received consulting compensation for Amgen, and honoraria from Arena Pharmaceuticals and Sunovian. Dr. G.A.L. has consulted for Astellas, Boehringer-Ingelheim, Dart Neuroscience, Heptares, Lundbeck, Merck, NeuroSig, and Takeda unrelated to this work. Drs. A.W.B., M.L.T., and M.T., as well as Ms. A.L.S. and Ms. S.Y.R. report no extra funding sources.

\section{Author details}

'VISN-22 Mental IIIness Research Education and Clinical Center (MIRECC), VA San Diego Healthcare System, San Diego, USA. ${ }^{2}$ Department of Psychiatry, University of California, San Diego, USA

\section{Conflict of interest}

The authors declare that they have no conflict of interest.

\section{Publisher's note}

Springer Nature remains neutral with regard to jurisdictional claims in published maps and institutional affiliations.

Supplementary Information accompanies this paper at https://doi.org/ 10.1038/s41398-018-0127-5.

Received: 26 June 2017 Revised: 24 October 2017 Accepted: 13 November 2017

Published online: 12 April 2018

\section{References}

1. Green, M. F. What are the functional consequences of neurocognitive deficits in schizophrenia? Am. J. Psychiatry 153, 321 (1996).

2. Braff, D. L. \& Light, G. A. Preattentional and attentional cognitive deficits as targets for treating schizophrenia. Psychopharmacology 174, 75-85 (2004).

3. Light, G. A. \& Swerdlow, N. R. Future clinical uses of neurophysiological biomarkers to predict and monitor treatment response for schizophrenia. Ann. N. Y. Acad. Sci. 1344, 105-119 (2015).

4. Moore, H., Geyer, M. A., Carter, C. S. \& Barch, D. M. Harnessing cognitive neuroscience to develop new treatments for improving cognition in schizophrenia: CNTRICS selected cognitive paradigms for animal models. Neurosci. Biobehav. Rev. 37, 2087-2091 (2013).

5. Thomas, M. L. et al. Modeling deficits from early auditory information processing to psychosocial functioning in schizophrenia. JAMA Psychiatry $\mathbf{7 4}$, 37-46 (2017).

6. Young, J. W. et al. Reverse translation of the rodent 5C-CPT reveals that the impaired attention of people with schizophrenia is similar to scopolamineinduced deficits in mice. Transl. Psychiatry 3, e324 (2013).

7. Sarter, M. Animal cognition: defining the issues. Neurosci. Biobehav. Rev. 28, 645-650 (2004).

8. Young, J. W. \& Geyer, M. A. Developing treatments for cognitive deficits in schizophrenia: the challenge of translation. J. Psychopharmacol. 29, 178-196 (2015).

9. Cope, Z. A., Powell, S. B. \& Young, J. W. Modeling neurodevelopmental cognitive deficits in tasks with cross-species translational validity. Genes Brain Behav. 15, 27-44 (2016).

10. Young, J. W., Light, G. A., Marston, H. M., Sharp, R. \& Geyer, M. A. The 5-choice continuous performance test: evidence for a translational test of vigilance for mice. PLOS ONE 4, e4227 (2009).

11. Lustig, C., Kozak, R., Sarter, M., Young, J. W. \& Robbins, T. W. CNTRICS final animal model task selection: control of attention. Neurosci. Biobehav. Rev. 37, 2099-2110 (2013).
12. van Enkhuizen, J. et al. Sleep deprivation impairs performance in the 5-choice continuous performance test: similarities between humans and mice. Behav. Brain Res. 261, 40-48 (2014).

13. MacQueen, D. A., et al. Amphetmaine improves human and mouse 5C-CPT performance. (in preparation).

14. Young, J. W. et al. Neurophysiological characterization of attentional performance dysfunction in schizophrenia patients in a reverse-translated task. Neuropsychopharmacology 42, 1338-1348 (2016).

15. Cornblatt, B. A. \& Keilp, J. G. Impaired attention, genetics, and the pathophysiology of schizophrenia. Schizophr. Bull. 20, 31-46 (1994).

16. Nuechterlein, K. H. Vigilance in schizoprhenia and related disorders. In: S. R. Steinhauer, J. H. Gruzelier, J. Zubin (eds). Neuropsychology, pscyhophysiology, and information processing. Hanbook of schizophrenia. 5, (397-433. Elsevier Science, New York, 1991).

17. Silverstein, S. M., Light, G. \& Palumbo, D. R. The sustained attention test: a measure of attentional disturbance. Comput. Human. Behav. 14, 463-475 (1998).

18. Nuechterlein, K. H., Dawson, M. E. \& Green, M. F. Information-processing abnormalities as neuropsychological vulnerability indicators for schizophrenia. Acta Psychiatr. Scand. 90, 71-79 (1994). s384.

19. Nuechterlein, K. H. et al. Attention/vigilance in schizophrenia: performance results from a large multi-site study of the Consortium on the Genetics of Schizophrenia (COGS). Schizophr. Res. 163, 38-46 (2015).

20. Barch, D. M., Moore, H., Nee, D. E., Manoach, D. S. \& Luck, S. J. CNTRICS imaging biomarkers selection: Working memory. Schizophr. Bull. 38, 43-52 (2011).

21. Conners, C. K. Conners' Continuous Performance Test (CPT-2) computer program for windows, technical guide, and software manual. (Multi Health Systems Inc, Toronto, ON, 2000).

22. Finkelstein, J. R., Cannon, T. D., Gur, R. E., Gur, R. C. \& Moberg, P. Attentional dysfunctions in neuroleptic-naive and neuroleptic-withdrawn schizophrenic patients and their siblings. J. Abnorm. Psychol. 106, 203 (1997).

23. Rutschmann, J., Cornblatt, B. \& Erlenmeyer-Kimling, L. Sustained attention in children at risk for schizophrenia: Report on a Continuous Performance Test. Arch. Gen. Psychiatry 34, 571-575 (1977).

24. Franke, P., Maier, W., Hardt, J., Hain, C. \& Cornblatt, B. A. Attentional abilities and measures of schizotypy: their variation and covariation in schizophrenic patients, their siblings, and normal control subjects. Psychiatry Res. 54, 259-272 (1994).

25. Chen, W. J. et al. Sustained attention deficit and schizotypal personality features in nonpsychotic relatives of schizophrenic patients. Am. J. Psychiatry 155, 1214-1220 (1998).

26. McGrew, K. S. CHC theory and the human cognitive abilities project: Standing on the shoulders of the giants of psychometric intelligence research. Intelligence 37, 1-10 (2009).

27. McCleery, A. et al. Latent structure of cognition in schizophrenia: a confirmatory factor analysis of the MATRICS Consensus Cognitive Battery (MCCB). Psychol. Med. 45, 2657-2666 (2015).

28. Andreasen, N. C. Scale for the Assessment of Negative Symptoms (SANS). (University of lowa, lowa City, 1983).

29. Andreasen, N. C. Scale for the assessment of positive symptoms (SAPS). (University of lowa, lowa City, 1984).

30. Lystad, J. U. et al. The MATRICS Consensus Cognitive Battery (MCCB): performance and functional correlates. Psychiatry Res. 220, 1094-1101 (2014).

31. Cornblatt, B. A., Risch, N. J., Faris, G., Friedman, D. \& Erlenmeyer-Kimling, L. The Continuous Performance Test, identical pairs version (CPT-IP): I. New findings about sustained attention in normal families. Psychiatry Res. 26, 223-238 (1988).

32. Cohen J., Cohen P., West S. G., Aiken L. S. Applied multiple regression/correlation analysis for the behavioral sciences. Routledge; Mahwah, New Jersey, USA, 2013.

33. Blanchard, J. J. \& Cohen, A. S. The structure of negative symptoms within schizophrenia: implications for assessment. Schizophr. Bull. 32, 238-245 (2005).

34. Sayers, S. L., Curran, P. J. \& Mueser, K. T. Factor structure and construct validity of the Scale for the Assessment of Negative Symptoms. Psychol. Assess. 8, 269 (1996).

35. Bismark, A. W. et al. Relationship between efforfful motivation and neurocognition in schizophrenia. Schizophr. Res. 193, 69-76 (2017).

36. Kahn, P. V. et al. Standardizing the use of the Continuous Performance Test in schizophrenia research: a validation study. Schizophr. Res. 142, 153-158 (2012). 
37. Lexén, A. \& Bejerholm, U. Occupational engagement and cognitive functioning among persons with schizophrenia: an explorative study. Scand. J. Occup. Ther. 9, 1-8 (2017).

38. Lee, R. S. et al. Neuropsychological and functional outcomes in recent-onset major depression, bipolar disorder and schizophrenia-spectrum disorders: a longitudinal cohort study. Transl. Psychiatry 5, e555 (2015).

39. van Erp, T. G. et al. Neuropsychological profile in adult schizophrenia measured with the CMINDS. Psychiatry Res. 230, 826-834 (2015).

40. Lee, K. H. et al. Performance on the continuous performance test under parametric increase of working memory load in schizophrenia. Psychiatry Res. 197, 350-352 (2012).

41. Hahn, B. et al. Visuospatial attention in schizophrenia: deficits in broad monitoring. J. Abnorm. Psychol. 121, 119 (2012).

42. Young, J. W. et al. GlyT-1 inhibition attenuates attentional but not learning or motivational deficits of the Sp4 hypomorphic mouse model relevant to psychiatric disorders. Neuropsychopharmacology 40, 2715-2726 (2015).
43. Barnes, S. A., Young, J. W. \& Neill, J. C. D1 receptor activation improves vigilance in rats as measured by the 5-choice continuous performance test. Psychopharmacology 220, 129-141 (2012).

44. Turner, K. M., Young, J. W., McGrath, J. J., Eyles, D. W. \& Burne, T. H. Cognitive performance and response inhibition in developmentally vitamin D (DVD)deficient rats. Behav. Brain Res. 242, 47-53 (2013).

45. McTeague, L. M. et al. Identification of common neural circuit disruptions in cognitive control across psychiatric disorders. Am. J. Psychiatry 174, 676-685 (2017).

46. de Leon, J. \& Diaz, F. J. A meta-analysis of worldwide studies demonstrates an association between schizophrenia and tobacco smoking behaviors. Schizophr. Res. 76, 135-157 (2005).

47. Reed, A. C., Harris, J. G. \& Olincy, A. Schizophrenia, smoking status, and performance on the matrics Cognitive Consensus Battery. Psychiatry Res. 246, 1-8 (2016). 